\title{
Perceptions of on-farm emergency slaughter for dairy cows in British Columbia
}

\author{
Katherine E. Koralesky* and David Fraser \\ Animal Welfare Program, Faculty of Land and Food Systems, University of British Columbia, Vancouver, V6T 1Z4, Canada
}

\begin{abstract}
Some jurisdictions permit on-farm emergency slaughter (OFES) as one end-of-life option for dairy cows and other animals that cannot be transported humanely but are deemed fit for human consumption. Anecdotal reports suggest that OFES is controversial among dairy industry professionals, but to date their perceptions of OFES have not been studied systematically. Twentyfive individual interviews and 3 focus groups with 40 dairy producers, veterinarians, and other professionals in British Columbia, Canada, revealed positive and negative perceptions of OFES influenced by (1) individual values, (2) the perceived operational legitimacy of OFES, and (3) concern over social responsibility and public perception of the dairy industry. Study participants valued cow welfare but were divided on whether OFES quickened or delayed death for injured animals. Views on the operational legitimacy of OFES varied because of different perceptions and concerns regarding regulatory, veterinary, and meat inspector oversight, a possible conflict of interest for veterinarians, and concerns over carcass hygiene and transport. Whereas many appreciated that OFES prevented transport of compromised cows, others saw OFES as merely a stopgap measure. Seven recommended actions could address concerns while retaining the benefits of OFES: (1) specifying precise timing parameters for OFES, (2) clarification of allowable cow conditions for OFES, (3) consultation with dairy industry professionals if OFES is to be expanded, (4) more proactive culling and the development of euthanasia protocols on farms, (5) the designation of veterinarians as the first point of contact in the OFES process, (6) veterinarian training on animal inspection and allowable conditions for OFES, and (7) the use of proper procedures and equipment during the OFES process to ensure food safety.
\end{abstract}

Received March 23, 2018.

Accepted September 7, 2018.

*Corresponding author: katie.koralesky@alumni.ubc.ca
Key words: dairy cow welfare, emergency slaughter, perceptions, culling decisions

\section{INTRODUCTION}

On-farm emergency slaughter (OFES) refers to the inspection, stunning, and bleeding of an animal on the farm before the carcass is transported to a slaughterhouse for sale. The stated goals of OFES are to avoid undue suffering of an injured animal and to salvage meat. On-farm emergency slaughter is a regulated option in the European Union and several Canadian provinces but is not allowed in the United States. In British Columbia, Canada, OFES is regulated by the provincial Meat Inspection Regulation (Government of British Columbia, 2014), and guidance documents are available (BCMA, 2014a, undated). By regulation, an animal may undergo OFES if (1) it "is in a physical condition that precludes it from being transported to a slaughter establishment without undue suffering," or (2) if the animal "poses a high risk of significant injury to humans if it is transported to a slaughter establishment." Guidance documents include information about, for example, antemortem inspections, humane stunning, transport hygiene, and certain diseases and conditions that exclude animals from the program including chronic conditions (BCMA, 2014a, undated).

In British Columbia and elsewhere, the OFES process requires coordination between dairy industry professionals, including producers, transporters, veterinarians, and meat inspectors. To use OFES, a producer must confirm that the slaughterhouse can accept the carcass and then a veterinarian must conduct an antemortem inspection (BCMA, 2014b) on the farm to confirm that the animal is fit for human consumption (i.e., no signs of disease). A transporter with a Specified Risk Material permit then stuns the animal (using a firearm) and bleeds it on the farm and transports the carcass to the slaughterhouse within $2 \mathrm{~h}$, where postmortem inspection is done by a meat hygiene inspector (BCMA, 2014a). On-farm emergency slaughter is currently available Monday through Friday. The dairy producer is responsible for paying veterinarian and transporter fees 
and the dairy producer would then receive a check from the slaughterhouse for the amount of meat salvaged. In contrast to OFES, on-farm euthanasia may be performed by farm staff or a deadstock collector. In that case, the producer would be responsible for composting the carcass or paying carcass disposal fees, which can range from Can $\$ 100$ to $\$ 200$.

Of the limited research on how industry professionals perceive OFES, most focuses on veterinarian challenges and views. In one study, Irish stakeholders reported a conflict between a veterinarian's professional duty to protect animal welfare and their client's desire to salvage the financial value of animals through OFES (Magalhães-Sant'Ana et al., 2017). In another study, $89 \%$ of veterinarians working in bovine slaughterhouses in Ireland did not want to accept OFES carcasses, citing food safety risks and decreased meat quality (McDermott and McKevitt, 2016). However, OFES can help prevent unassisted on-farm dairy cow mortality, which involves financial loss (Alvåsen et al., 2014) and is a concern to dairy industry professionals because of animal welfare implications (Ventura et al., 2015).

On-farm emergency slaughter often occurs in situations where dairy industry professionals are faced with a decision that is unexpected and unwanted, and where there may be uncertainty over the diagnosis of the condition and prognosis for the cow. In these situations, the welfare of the individual animal is compromised. Whereas anecdotal reports suggest OFES is controver- sial, little is known about how it is perceived by the individuals who are involved. These perceptions likely influence the use and coordination of the program and can also provide insight into whether the goals of OFES are met. Our study used semistructured individual interviews and focus groups (1) to understand the concerns and perceived benefits that influence how OFES is regarded, understood, and interpreted, and (2) to analyze these different views to develop recommendations for OFES.

\section{MATERIALS AND METHODS}

This study was approved by the University of British Columbia Behavioral Research Ethics Board. A semistructured interview guide (Table 1) was designed to explore participants' experience with, and perceptions of, OFES. A pilot study of 4 interviews and 1 focus group (8 participants) was conducted in spring 2016 to confirm that the interview guide was effective in reaching study objectives.

In summer 2016, the study continued using sampling criteria that required participants to be dairy industry professionals in British Columbia familiar with OFES. On-farm emergency slaughter typically occurs in southwestern British Columbia, where many dairy farms are located in the vicinity of a slaughterhouse that accepts OFES carcasses. A script was used via email, telephone, or in person to introduce the study

Table 1. Semistructured interview guide

\begin{tabular}{|c|c|c|}
\hline Theme & Primary questions & Follow-up questions \\
\hline $\begin{array}{l}\text { General experience with } \\
\text { on-farm emergency } \\
\text { slaughter (OFES) }\end{array}$ & Tell me about your involvement with OFES. & $\begin{array}{l}\text { Does your role vary? } \\
\text { Can you describe any specific examples of how } \\
\text { you have been involved with or exposed to the } \\
\text { program? }\end{array}$ \\
\hline $\begin{array}{l}\text { General perceptions } \\
\text { of OFES }\end{array}$ & $\begin{array}{l}\text { What have you heard about the program? } \\
\text { What do you feel are the positive aspects of this } \\
\text { program? } \\
\text { What do you feel are the negative aspects of this } \\
\text { program? } \\
\text { Why do supporters support the program? } \\
\text { Why do opponents oppose the program? }\end{array}$ & \\
\hline $\begin{array}{l}\text { Outcomes for dairy } \\
\text { industry professionals }\end{array}$ & $\begin{array}{l}\text { How does this program affect: } \\
\text {-veterinarians } \\
\text {-producers } \\
\text {-meat inspectors } \\
\text {-other dairy industry professionals? }\end{array}$ & \\
\hline \multirow[t]{2}{*}{$\begin{array}{l}\text { Outcomes for dairy } \\
\text { cattle }\end{array}$} & $\begin{array}{l}\text { One of the goals of this program is to prevent animal } \\
\text { suffering. Is OFES achieving this goal? }\end{array}$ & Why or why not? \\
\hline & $\begin{array}{l}\text { Does this program currently have the ability to improve } \\
\text { the welfare of dairy cattle, especially at the end of their } \\
\text { lives? }\end{array}$ & $\begin{array}{l}\text { Does OFES have any unrealized potential? Is } \\
\text { there anything that it could do but is not yet } \\
\text { achieving? }\end{array}$ \\
\hline \multirow[t]{2}{*}{$\begin{array}{l}\text { Final thoughts and } \\
\text { recommendations }\end{array}$} & $\begin{array}{l}\text { What in your work has changed as a result of the } \\
\text { implementation of OFES? }\end{array}$ & \\
\hline & $\begin{array}{l}\text { Would you recommend that other provinces or } \\
\text { jurisdictions adopt OFES? If yes or no, what changes } \\
\text { would you recommend? }\end{array}$ & Is there anything else you'd like to add? \\
\hline
\end{tabular}


to participants. Sampling methods included purposive and snowball or referral sampling (Miles et al., 2014). Purposive sampling was used to select participants known to play specific roles in OFES and to select veterinary clinics known to participate in OFES. Snowball or referral sampling, whereby existing or known participants identify or help to recruit future participants, was done through veterinarians who recruited their clients to participate. Participants who use and do not use OFES were recruited. Those who agreed to participate contacted the researcher directly or consented that their contact information be given to the researcher. The researcher had no access to private participant information other than name and contact details. Each participant gave written consent before the interview or focus group began. Each interview and focus group was audio recorded.

Twenty-five interviews and 3 focus groups (including the pilot study) were conducted with 40 participants (35 men and 5 women). Twenty-four participants were dairy producers, 12 were large animal (dairy) veterinarians, and 4 were other dairy industry professionals playing roles in the OFES process. The 3 focus groups involved 2, 4, and 5 veterinarians, respectively, in each case from the same veterinary clinic. A focus group format was used to stimulate discussion among colleagues to provide more depth of insight, but this was not possible for the other participants. Herd size of producers ranged from 70 to 700 cows, spanning the average herd size in British Columbia of approximately 180 cows in the year of the study (Canadian Dairy Information Centre, 2017). All producers used loosehousing systems as is typical of British Columbia dairy farms (Canadian Dairy Information Centre, 2017). All participants lived in the southwestern region of British Columbia, where approximately $75 \%$ of British Columbia's milk is produced (BCMA, 2014c), except for one veterinarian and one producer who lived on Vancouver Island, where approximately 14\% of British Columbia's milk is produced (BCMA, 2014c).

Interviews lasted from 14 to $64 \mathrm{~min}$ (mean of $33 \mathrm{~min}$ ) and focus groups from 52 to $66 \mathrm{~min}$ (mean of $59 \mathrm{~min}$ ). Interviews and focus groups were conducted by the first author at the participant's home (20), office (3), at a location of the participant's choosing (1), or on the telephone (4). The number of interviews and focus groups was not predetermined; rather, the study continued until data saturation was reached. Data saturation is the point when comments raised are a repetition of comments raised previously by other participants (Guest et al., 2006).

Interviews and focus groups were transcribed and checked for accuracy by the researcher. Before data analysis began, each participant received a copy of their interview or focus group transcript and was given $2 \mathrm{wk}$ to ensure that it accurately represented what they intended to convey (Miles et al., 2014). No modifications were requested. Each participant was given a unique identifier that included a letter designating their professional group $(\mathrm{p}=$ producer, $\mathrm{v}=$ veterinarian, $\mathrm{o}=$ other industry professional) and 3 random numbers.

Initial line-by-line coding, a process of labeling segments of text with codes (words or short phrases), was used to analyze each transcript (Charmaz, 2006). Applied thematic analysis (Guest et al., 2012) was then used to develop themes by grouping similar codes that categorize participants' perceptions of OFES. Intercoder agreement, a process whereby researchers analyze the same data and compare and discuss results (Guest et al., 2012), was used first in the early coding process to determine initial codes and second after the first author had coded each transcript and produced a codebook. This codebook was then used by another trained researcher to code a sample of transcripts. In each instance, coding discrepancies were discussed and resolved.

\section{RESULTS}

Perceptions of OFES were both positive and negative and ranged from seeing no drawbacks of OFES to questioning its necessity. These perceptions, which appeared to affect decisions on whether to use OFES, were heavily influenced by 3 major themes: (1) individual values, (2) the perceived operational legitimacy of OFES, and (3) concerns over social responsibility and public perception of the dairy industry.

\section{Individual Values}

Perceptions of OFES were influenced by the value that individuals attached to cow welfare, financial gain, and meat salvage. All participants indicated that they value cow welfare, but they made different decisions about using OFES. Some participants believed that OFES promotes fast decision-making and thus reduces the delay in slaughtering compromised animals. As V913 stated: "... some farms might be quicker to slaughter these animals than try to rehabilitate them, when really [there] wasn't a lot of hope." In this and other cases, OFES was perceived to be positive for cow welfare because it decreases the amount of time that a cow may suffer in transport or during unsuccessful rehabilitation on the farm.

In contrast, other participants believed that OFES is negative for cow welfare because it prolongs animal suffering, for example, if the unavailability of the veterinarian, transporter, or slaughterhouse causes delays 
in slaughtering a cow with a traumatic injury. This was articulated by P342: "If I have an animal in distress, I'm not waiting for the whole thing to get coordinated and someone to show up."

More generally, some participants recognized that rapid decision-making for compromised cows had a large impact on animal welfare. Using a hypothetical situation, V255 pondered: "If you had a cow that dislocated her leg or did something on a Thursday night, and that has to wait until the following morning at seven - that's several hours of pain and discomfort. Would the farmer have gone and euthanized the cow with his own gun or phoned a deadstock guy to come and pick up that animal before seven? Maybe, maybe not."

Although some participants mentioned financial gain from OFES (participants reported an average Can $\$ 200$ per cow), others saw it more as a way to prevent the cost of carcass disposal. As P891 stated: "Revenue-neutral is fine ... I'm not doing it for the money, right?"

On-farm emergency slaughter was also seen as a positive opportunity to salvage meat from an injured animal that was "perfectly healthy" (P280), had "done her job [produced milk]" (P350), and could be used for "feeding somebody" (P919) instead of being wasted. This was especially important to participants because of the time, resources, and effort they put into raising animals, as noted by P932: “... psychologically, it's just a lot better, as a farmer, when you know that ... I've put a lot of work into this cow. She's been a good cow and now she's going to go for meat ... Everything's good about her except she hurt her knee ... It's just easier for a farmer to know that it's not being wasted."

\section{Operational Legitimacy of OFES}

Certain operational aspects of OFES influenced how participants perceived the legitimacy of the program by either building confidence or causing concern. These aspects include the manner in which OFES was originally implemented, logistics of OFES (including veterinarianproducer relationships), oversight of OFES, and food safety concerns.

Participants expressed that the purpose and appropriate use of OFES could have been made clearer when the program was first implemented. O284 expressed this sentiment: "... [OFES] was a learning curve ... What kind of animals fit the program? ... Where do you draw the line?" Veterinarians also noted a lack of clarity and communication about acceptable animal conditions for OFES and felt they had to rely on their judgement about what was acceptable. As V319 stated: "... we're not truly trained in premortem inspections for slaughter... so it's hard for us to make that call." Although guidance documents state that chronic con- ditions are not an acceptable reason for OFES, participants were unclear on how this applied. As V913 pondered, "... [there's] a cow that's been lame for a while, [a farmer] decides 'Well, we can't transport her,' but is that a chronic condition?" Additionally, most participants stated that they learned about OFES by word of mouth or in unofficial ways. As V255 reported: "One of our clients knew about it before we did"; thus, a lack of clear guidelines and communication at the outset appears to have decreased confidence in the legitimacy of OFES.

Participants were divided in their level of confidence in the logistics of OFES. Some considered it a simple process that is "an easy option" (P505), "a program that's accessible" (P686), and a process that "takes fifteen, twenty minutes, and then the cow leaves" (V262). Other participants, however, expressed concerns about the time constraints of OFES based on the availability of the slaughterhouse, the veterinarian, and the transporter. Additionally, the amount of coordination and paperwork, although perceived to be necessary, made OFES inconvenient and increased concerns that OFES could delay the death of injured cows. As P477 summarized: "You got to get the vet out, and then it's the time factor to actually shoot and bleed an animal, to then transport her. And so sometimes it's kind of like, 'Is it worth all of this?'" For others, initial apprehension about OFES waned as time passed. When asked how dairy industry professionals perceive OFES, O202 stated: "People seem to have kind of gone on board... it seems as though it must be working - I guess very well - or else it wouldn't continue. We're three years in... obviously it's working fairly well."

The order of events during the OFES process also created concern. On-farm emergency slaughter guidelines state that a producer must first verify that the slaughterhouse can receive the carcass and then a veterinarian must perform an antemortem inspection. However, when asked to describe the OFES process, some participants stated that the veterinarian would be telephoned first, whereas others would first telephone the transporter. In the latter case, veterinarians may feel pressured or obligated to approve OFES, as V262 explained: "we're making that call when the truck's there and the rifle's loaded." Thus, it appears that veterinarians may feel conflict if they are not the first point of contact for OFES, are not given adequate time to assess the cow, or if they feel they are being asked to simply endorse a decision that the producer has already made.

Nonetheless, veterinary, regulatory, and meat inspection oversight were perceived to increase the legitimacy of OFES. Some participants expressed confidence in their veterinarian's ability to perform inspections; 
as P280 stated: "The veterinarian has to come out and check everything ... he has to approve it first." Participants gained additional confidence due to the perceived oversight from regulatory requirements and meat inspection at the slaughterhouse. As P138 stated: "It's just more regulated, right? You have ... a set parameter of what cows can be in there, a set time of how long it takes to get to the kill plant. I know it's very strict."

Finally, participants shared food safety concerns related to transport and carcass cleanliness. In contrast to regular slaughter, OFES is often used for nonambulatory animals (Koralesky and Fraser, 2018). Although transport and carcass hygiene is covered in the OFES guidance documents, some participants still expressed concern over carcass cleanliness. As V913 recalled: "I've seen these [freshly killed] animals that are dragged through manure ... they are completely covered with manure. How do you possibly skin these animals cleanly and effectively without contaminating the meat underneath?"

In summary, some participants were confident in the various levels of oversight of OFES and appreciated that OFES is an option for cows. For others, a perception that program implementation lacked communication and clarity, combined with food safety concerns, decreased confidence in the program. In addition, participants were divided in their perception of whether the process and logistics of OFES made it quick and easy to use or too complicated and inconvenient.

\section{Social Responsibility and Public Perception of the Dairy Industry}

Finally, participants expressed concern over social responsibility and public perception of the dairy industry. Some participants, noting that the public is largely unaware of how dairy farms and auction markets operate, expressed concern that OFES could be misinterpreted and lead to negative publicity spread via the media and social media. In contrast, other participants considered that OFES reduces the scope for negative publicity by preventing live compromised cows from being seen on trucks and at auction markets. Auction markets are open to the public; hence, many participants, such as P517, believed: "If you can eliminate these marginal animals going to market, I think it's just better all around for the industry."

Nonetheless, some participants saw OFES as a stopgap measure. Proactive culling was discussed as a more appropriate and long-term solution that could be used to decrease OFES and avoid situations where unwanted and unexpected decisions have to be made. As P350 reasoned: "If they're getting too old, we'll look at her and say, 'Why would we rebreed that cow? Her legs are falling apart. Her udder is not good. She's done her job. She's in good health. She still can be used for consumable products...' The whole thing is trying to [be] preventative, before we get there ... For us it's a lot less stress if we cull on time because we don't have these animals that we know are potentially going to be problems." Thus, participants appreciated that management practices such as proactive culling could decrease the number of injuries on farms and potentially reduce future problems.

Participants also discussed the industry's responsibility for food safety. Some noted that their decision to use OFES for a cow was often guided by asking, 'Would I eat it?' Veterinarians also found this question helpful when discussing OFES candidates with their clients. As V262 stated: "Without examining the cow, I'd say, 'Would you eat her?' And if they say 'no' we'd both agree that, okay, let's not send it, regardless of why she's down or how long she was down. If the farmer and I can look at each other honestly and say we wouldn't eat this, how can we expect someone else to?"

Finally, some participants expressed concern that the option to gain financially from an injured or compromised animal could unduly affect management decisions. For example, P653 expressed concern that producers might delay OFES until a drug withdrawal period passed, noting that OFES "shouldn't be a tool to fix poor management."

In summary, participants' perceptions of OFES were clearly influenced by concerns over public perception of the dairy industry and responsible management of compromised cows. Inasmuch as it reduces the transport of compromised cows, participants perceived OFES as a positive option for special circumstances, but not as a routine or fully satisfactory means of managing compromised cows.

\section{DISCUSSION}

The following discussion uses shared values and concerns identified in the study to propose recommendations that could help address negative perceptions of OFES while retaining its advantages.

In our study, all participants valued cow welfare, but this led some participants to use OFES in the belief that it promotes fast decision-making for compromised cows, whereas others refused to use the program in the belief that it extends animal suffering. The shared goal of minimizing both cow suffering and delays could be reached through 2 recommendations for OFES. First, precise timing parameters are needed for OFES so that significant delays from injury to slaughter are avoided. Second, noting that chronic conditions such as lameness 
were included as a reason for OFES on antemortem inspection documents (Koralesky and Fraser, 2018), clarification of which conditions are allowable for OFES is necessary. In addition, producers and veterinarians need information about the likelihood of recovery for nonambulatory cows (see Green et al., 2008) and the importance of good nursing care (Poulton et al., 2016; Stojkov et al., 2016) in cases where the prognosis is unclear.

Our study illustrates that the legitimacy of a program can be influenced by both its outcomes and how it was developed. Bradley and MacRae (2011), in identifying features that give legitimacy to a program, examined the process for developing codes of practice used by Canada's National Farm Animal Care Council. Those authors defined legitimacy as whether stakeholders "consider the regulatory body or network developing the rules to be authoritative, to use right process, [and] to be adding value." Further, they divide legitimacy factors into inputs (how the program was developed, whether stakeholder representation was present) and outputs (whether the program or policy is ultimately perceived as effective). In our study, some participants perceived a poor implementation process and lack of initial communication, which reduced input legitimacy. Despite this, many participants expressed confidence in the program outcomes and some of the initial apprehension waned as OFES continued over time, indicating perceived output legitimacy at least for some participants. Hence, a third recommendation is that the dairy industry consider the necessary inputs and outputs during the development of future programs and policies and consult with dairy industry professionals if OFES is to be expanded in other parts of the province.

Participants were sensitive to public perception of their industry and feared that the public may misinterpret OFES because of a lack of awareness of farming practices (see Benard and de Cock Buning, 2013). Others appreciated that OFES reduces the chance that the public may see and photograph compromised cows at public auction. Participants often referred to previous well-publicized undercover videos that reduced public confidence and have been shown to increase negative attitudes toward animal agriculture (Tiplady et al., 2013). Nonetheless, some participants saw OFES as a stopgap measure and felt that it should be used only in exceptional circumstances and not to compensate for poor management. Therefore, a fourth recommendation is that individual farms prioritize compromised cow management through proactive culling and the development of euthanasia protocols. Such protocols should include information about making timely decisions for compromised animals as well as clear delegation of decision-making among farm staff. Proactive culling could reduce the need to use OFES as well as other animal welfare problems (NFAHWC, 2017). Euthanasia protocols can help provide clarity in situations where diagnosis and prognosis of injured cows is uncertain (Turner and Doonan, 2010; Poulton et al., 2016), and they are increasingly required by on-farm animal welfare assessment programs.

Veterinarian-producer relationships may be affected by OFES because the veterinarian is required to perform an antemortem inspection before OFES is used. On the one hand, producers held veterinary oversight in high regard. On the other hand, veterinarians and producers saw a possible conflict of interest in OFES cases, for example, if a veterinarian felt obligated to approve a cow for OFES. Magalhães-Sant'Ana et al. (2017) found a similar conflict reported by focus groups with Irish stakeholders. In British Columbia, this conflict and pressure to approve cows for OFES may be increased in cases where the transporter is contacted before the veterinarian is called. Hence, a fifth recommendation is that veterinarians be clearly designated as the first point of contact for confirming cow eligibility for OFES. This could potentially eliminate any sense of obligation felt by veterinarians to endorse a producer's decision.

Some veterinarians indicated that they did not feel comfortable performing antemortem inspections of cows without training. In Alberta, veterinarians receive specific training on OFES before they are allowed to perform the procedure (Government of Alberta, 2017), and certain veterinarians in Ontario are designated to perform OFES inspections (Ontario Ministry of Agriculture, Food and Rural Affairs, 2018). The implementation of similar requirements in British Columbia could clarify gray areas of allowable cow conditions and help eliminate a sense of obligation that may be felt by veterinarians who have relationships with their clients. Therefore, a sixth recommendation is that veterinarians receive specific training on OFES, much as recommended by Magalhães-Sant'Ana et al. (2017).

Finally, food safety concerns were mostly related to carcass cleanliness, and thus a seventh recommendation is to ensure clean conditions for bleeding and transport, and possibly a refrigerated vehicle, which could improve the perceived operational legitimacy of OFES.

\section{CONCLUSIONS}

Participants perceived OFES in positive and negative ways based on their individual values, their perceptions of the legitimacy of OFES, and concern over social responsibility and public perception of the dairy industry. Participants valued cow welfare in different ways that resulted in variation of OFES use. Some saw OFES as 
a stopgap measure rather than a satisfactory solution to compromised cow management. Recommendations such as the creation of precise timing parameters and clarification on acceptable cow conditions for OFES may help alleviate concerns regarding timing delays and inappropriate use of the program. The development and use of proactive culling and euthanasia protocols on farms could facilitate good end-of-life decision-making in uncertain situations.

\section{ACKNOWLEDGMENTS}

We thank each participant in this study. We also thank the Loblaw Companies Limited (Brampton, Ontario, Canada) and the University of British Columbia Animal Welfare Program for funding. Finally, we thank the reviewers for their helpful comments and suggestions.

\section{REFERENCES}

Alvåsen, K., P. T. Thomsen, C. Hallén Sandgren, M. Jansson Mörk, and U. Emanuelson. 2014. Risk factors for unassisted on-farm death in Swedish dairy cows. Anim. Welf. 23:63-70. https://doi org/10.7120/09627286.23.1.063.

BCMA. 2014a. Guidelines for establishment operators and animal producers or agents: Emergency slaughter under the B.C. Meat Inspection Regulation. British Columbia Ministry of Agriculture. Accessed Mar. 19, 2018. https://www2.gov.bc.ca/assets/gov/farming -natural-resources-and-industry/agriculture-and-seafood/food -safety/meat-inspection/produceremergencyslaughterguidelines .pdf.

BCMA. 2014b. Document for an approved emergency slaughter on farm. British Columbia Ministry of Agriculture. Accessed Mar. 19, 2018. https://www2.gov.bc.ca/assets/gov/farming-natural -resources-and-industry/agriculture-and-seafood/food-safety/meat -inspection/emergencyslaughterfarmform.pdf.

BCMA. 2014c. Strengthening farming - right to farm: Commodity - dairy. Accessed Mar. 19, 2018. https://www2.gov.bc.ca/ assets/gov/farming-natural-resources-and-industry/agriculture -and-seafood/agricultural-land-and-environment/strengthening -farming/farm-practices/870218-7_dairy.pdf.

BCMA. Undated. Guidelines for veterinary practitioners: Emergency slaughter under the B.C. Meat Inspection Regulation. British Columbia Ministry of Agriculture. Accessed Mar. 19, 2018. https: //www2.gov.bc.ca/assets/gov/farming-natural-resources-and -industry/agriculture-and-seafood/food-safety/meat-inspection/ emergencyslaughterguidelines.pdf.

Benard, M., and T. de Cock Buning. 2013. Exploring the potential of Dutch pig farmers and urban-citizens to learn through frame reflection. J. Agric. Environ. Ethics 26:1015-1036. https://doi.org/ 10.1007/s10806-013-9438-y.

Bradley, A., and R. MacRae. 2011. Legitimacy \& Canadian farm animal welfare standards development: The case of the National Farm Animal Care Council. J. Agric. Environ. Ethics 24:19-47. https:// doi.org/10.1007/s10806-010-9240-z.
Canadian Dairy Information Centre. 2017. Dairy facts and figures. Accessed Mar. 19, 2018. http://www.dairyinfo.gc.ca/index_e.php ?s1 $=$ dff-fcil\&s $2=$ farm-ferme\&s $3=$ nb.

Charmaz, K. 2006. Constructing Grounded Theory: A Practical Guide Through Qualitative Analysis. SAGE Publications, London, UK.

Government of Alberta. 2017. Appointed Inspector Program. Accessed Mar. 19, 2018. http://www1.agric.gov.ab.ca/general/progserv.nsf/ all/pgmsrv456.

Government of British Columbia. 2014. Meat Inspections Regulation. B.C. Reg. 205/2014. Queen's Printer (QP), Victoria, British Columbia, Canada. Accessed Mar. 19, 2018. http://www.bclaws .ca/EPLibraries/bclaws_new/document/ID/freeside/349_2004 \#section14.

Green, A. L., J. E. Lombard, L. P. Garber, B. A. Wagner, and G. W. Hill. 2008. Factors associated with occurrence and recovery of nonambulatory dairy cows in the United States. J. Dairy Sci. 91:2275-2283. https://doi.org/10.3168/jds.2007-0869.

Guest, G., A. Bunce, and L. Johnson. 2006. How many interviews are enough?: An experiment with data saturation and variability. Field Methods 18:59-82. https://doi.org/10.1177/1525822X05279903.

Guest, G., K. M. MacQueen, and E. E. Namey. 2012. Applied Thematic Analysis. SAGE Publications, Thousand Oaks, CA.

Koralesky, K. E., and D. Fraser. 2018. Use of on-farm emergency slaughter for dairy cows in British Columbia. J. Dairy Sci. 101:6413-6418. https://doi.org/10.3168/jds.2017-14320.

Magalhães-Sant'Ana, M., S. J. More, D. B. Morton, and A. J. Hanlon. 2017. Challenges facing the veterinary profession in Ireland: 3. emergency and casualty slaughter certification. Ir. Vet. J. 70:24. https://doi.org/10.1186/s13620-017-0102-0.

McDermott, P., and A. McKevitt. 2016. Analysis of the operation of on farm emergency slaughter of bovine animals in the Republic of Ireland. Ir. Vet. J. 69:4. https://doi.org/10.1186/s13620-016-0063 -8 .

Miles, M. B., A. M. Huberman, and J. Saldaña. 2014. Qualitative Data Analysis: A Methods Sourcebook, 3rd ed. SAGE Publications, Thousand Oaks, CA.

NFAHWC. 2017. Cull dairy cow expert consultation: Consensus statement. National Farmed Animal Health and Welfare Council (NFAHWC). Accessed Mar. 19, 2018. http://www.ahwcouncil .ca/pdfs/NFAHW\%20Council_Cull\%20Cow\%20Consensus\%20 Document_2017_2.pdf.

Ontario Ministry of Agriculture, Food and Rural Affairs. 2018. Emergency slaughter. OMAFRA. Accessed Mar. 19, 2018. http://www .omafra.gov.on.ca/english/food/inspection/es.htm.

Poulton, P. J., A. L. Vizard, G. A. Anderson, and M. F. Pyman. 2016 High-quality care improves outcome in recumbent dairy cattle. Aust. Vet. J. 94:173-180. https://doi.org/10.1111/avj.12445.

Stojkov, J., D. M. Weary, and M. A. G. von Keyserlingk. 2016. Nonambulatory cows: Duration of recumbency and quality of nursing care affect outcome of flotation therapy. J. Dairy Sci. 99:20762085. https://doi.org/10.3168/jds.2015-10448.

Tiplady, C. M., D. A. B. Walsh, and C. J. Phillips. 2013. Public response to media coverage of animal cruelty. J. Agric. Environ. Ethics 26:869-885. https://doi.org/10.1007/s10806-012-9412-0.

Turner, P. V., and G. Doonan. 2010. Developing on-farm euthanasia plans. Can. Vet. J. 51:1031-1034.

Ventura, B. A., M. A. G. von Keyserlingk, and D. M. Weary. 2015. Animal welfare concerns and values of stakeholders within the dairy industry. J. Agric. Environ. Ethics 28:109-126. https://doi.org/10 .1007/s10806-014-9523-x. 